\title{
Filtraciones en bypass gástrico resectivo por laparotomía en obesidad mórbida*
}

\author{
Dra. ANA MARÍA BURGOS L. ${ }^{1}$ \\ 1 Departamento de Cirugía, Hospital Clínico Universidad de Chile. \\ Santiago, Chile.
}

TRABAJO DE INGRESO

\begin{abstract}
Anastomotic leaks after roux-en $Y$ gastric bypass for morbid obesity

Background: Obesity is a severe disease whose prevalence continues to increase. Resective gastric bypass is one of the surgical procedures used in our center in patients with obesity, but like other techniques is not without complications, and the leak is one of the most serious. Aim: To determine by a prospective study the incidence, clinical presentation, postoperative course and treatment of obese patients with leaks after open resective gastric bypass. Material and Methods: From August 1999 to December 2009, 900 morbidly obese patients underwent open resective Roux-en-Y gastric bypass at the Hospital of the University of Chile. The use of drains and leak test with methylene blue during surgery, such as barium x-ray were performed in all patients as part of a protocol. Clinical signs, development, testing and treatment were recorded. Results: 36 patients $(4 \%)$ developed leaks. Of the 900 patients died $3(0.33 \%)$. Fever and tachycardia were the predominant symptoms. The diagnosis was made by imaging studies in 19 cases $(52.7 \%)$. The leaks were mostly between 5 th and 9 th postoperative day. The most frequent location of the leak was at the gastrojejunal anastomosis (58.3\%). Treatment was medical in 24 patients $(66.7 \%)$, requiring re-operation only in 12 cases. The average time of closure of the leak was 35.5 days and the patients were hospitalized a total of 33.7 days on average. Conclusions: In the series studied after resective gastric bypass the leak is presented in $4 \%$ of patients. Fever and tachycardia are useful signs to suspect a leak. The early detection of leaks is determinant to reach the best evolution of the patient and success in the treatment.
\end{abstract}

Key words: Leak, complications, morbid obesity, gastric bypass, morbidity.

\section{Resumen}

Introducción: La obesidad es una enfermedad severa cuya prevalencia continúa en incremento. El bypass gástrico resectivo, es una intervención quirúrgica realizada en nuestro centro, para obesidad; al igual que otras técnicas no está exenta de complicaciones, siendo la filtración una de las más graves. Objetivos: Determinar mediante estudio prospectivo la incidencia, presentación clínica, evolución y tratamiento de pacientes obesos con filtraciones después de bypass gástrico resectivo vía abierta. Material y Método: Desde agosto de 1999 a diciembre de 2009, 900 obesos mórbidos fueron sometidos a bypass gástrico resectivo en Y de Roux vía abierta en el Hospital Clínico de la Universidad de Chile. Se realizó a los pacientes por protocolo, prueba con azul de metileno intraoperatoria y control radiológico con bario postoperatorio. Fue registrada la evolución, exámenes y tratamiento realizado. Resultados: 36 pacientes (4\%) desarrollaron filtraciones. De 900

* Recibido el 20 de junio de 2011 y aceptado para publicación el 25 de julio de 2011.

Correspondencia: Dra. Ana María Burgos L.

Santos Dumont 999, Santiago, Chile.

anamburgos@yahoo.com.mx 
pacientes fallecieron $3(0,33 \%)$. Fiebre y taquicardia fueron síntomas predominantes. El diagnóstico fue realizado por estudios radiológicos en 19 casos $(52,7 \%)$. Las filtraciones se produjeron mayormente entre el $5^{\circ}$ y $9^{\circ}$ día postoperatorio. Localización más frecuente de filtración fue la anastomosis gastroyeyunal $(58,3 \%)$. Tratamiento médico en 24 pacientes $(66,7 \%)$ y reoperación en 12 casos. Tiempo promedio de cierre de filtración 35,5 días y hospitalización 33,7 días. Conclusiones: En la serie, la filtración post bypass gástrico resectivo se presentó en $4 \%$ de pacientes. Fiebre y taquicardia son signos útiles para sospechar filtración. El diagnóstico precoz de las filtraciones es determinante en la mejor evolución del paciente y éxito en el tratamiento.

Palabras clave: Filtración, complicaciones, obesidad mórbida, bypass gástrico, morbilidad.

\section{Introducción}

La obesidad es una enfermedad crónica, severa, multifactorial y cuya prevalencia continúa en incremento. En el tratamiento quirúrgico de la obesidad, el bypass gástrico (BPG) laparotómico o laparoscópico, es muy efectivo en la baja de peso y mejoría de comorbilidades ${ }^{1-3}$. En nuestro hospital realizamos el BPG con resección gástrica por las patologías potenciales de la mucosa gástrica y la dificultad para evaluación futura por endoscopia del estómago excluido del tracto gastrointestinal ${ }^{4,5}$. En el BPG se reportan diversas complicaciones postoperatorias y una de ellas es la filtración de la anastomosis.

El objetivo es analizar la presentación y tratamiento de las filtraciones en obesos sometidos a BPG gástrico resectivo en nuestra institución.

\section{Material y Método}

\section{Pacientes}

Se incluyen 900 pacientes obesos mórbidos, operados con bypass gástrico resectivo (BPGR) vía abierta, entre el 9 de agosto de 1999 y el 31 de diciembre de 2009, por cirujanos del Depto. Cirugía y aplicándose la misma técnica quirúrgica.

698 fueron mujeres $(77,6 \%)$ y 202 hombres (22,4\%), promedio de edad 40,8 \pm 12 años, (14-64) e Índice de Masa Corporal (IMC) 43,5 $\pm 5,7 \mathrm{~kg} / \mathrm{m}^{2}$ (35-67,4).

\section{Procedimiento Quirúrgico}

En todos los pacientes se realizó BPGR en Y de Roux laparotómico, técnica previamente publicada ${ }^{4,5}$. Laparotomía mediana supraumbilical, formación de reservorio gástrico de $20 \mathrm{ml}$, sección gástrica a $4 \mathrm{~cm}$ del cardias por curvatura menor usando engrapadoras lineales cortantes GIA 60 y 80 (Covidien, Cincinnatti). Resección del segmento gástrico distal, dividiendo el duodeno con engrapadora lineal GIA 60. Gastroyeyuno-anastomosis con engrapadora circular $\mathrm{N}^{\circ} 25$ (Covidien), diámetro interno de $15 \mathrm{~mm}$ y refuerzo con sutura reabsorbible interrumpida (Byosin 4-0). Asa alimentaria de Y de Roux ascendida transmesocólica, longitud entre 150 - $200 \mathrm{~cm}$ según grado de obesidad. Prueba con azul de metileno, 20-30 cc por sonda nasogástrica, comprobando impermeabilidad de suturas y capacidad del reservorio gástrico. Dos drenes de silicona adyacentes a anastomosis gastroyeyunal y duodenal.

\section{Definición de términos}

Filtración es falla en la hermeticidad o abertura espontánea en anastomosis o sutura de bordes seccionados en un órgano. Se mencionan varios sinónimos como dehiscencia, disrupción, incompetencia e insuficiencia de anastomosis. El United Kingdom Surgical Infection Study Group (SISG), define filtración anastomótica como "salida de contenido luminal de una unión quirúrgica entre dos vísceras huecas $^{6,7}$.

\section{Diagnóstico de filtración}

Extravasación del contraste y/o demostración de colección intraabdominal en un estudio radiológico, o salida de contenido gastrointestinal o biliar por drenes.

\section{Clasificación de las filtraciones:}

1. Tiempo de aparición de filtración ${ }^{8,9}$ :

a. Temprana: $1^{\circ}-4^{\circ}$ día postoperatorio (DPO).

b. Intermedia: $5^{\circ}-9^{\circ}$ DPO.

c. Tardía: $\geq 10^{\circ} \mathrm{DPO}$.

2. Severidad clínica ${ }^{10}$.

a. Tipo I o subclínica: Filtración local, ningún derrame, a través de tracto fistuloso a cavidad pleural o abdominal o aparición de contraste en dren abdominal.

b. Tipo II o clínica: Filtración con gran diseminación a cavidad pleural o abdominal, por trayecto irregular, aparición de contraste en drenes abdominales.

\section{Evaluación Clínica}

Evaluación de síntomas y signos cada 6 horas. Registro de localización, método diagnóstico, tratamiento, tiempo de aparición y cierre de filtraciones.

\section{Control Radiológico}

Estudio gastrointestinal (GI) con sulfato de bario, entre $4^{\circ}$ y $5^{\circ} \mathrm{DPO}$, evaluándose tamaño reservorio gástrico y pasaje en anastomosis. 


\section{Análisis Estadístico}

Estudio prospectivo, descriptivo, aplicándose protocolo a cada paciente. Datos expresados como promedio, desviación estándar (D.E.) y rango. Prueba $t$ de student, significativo $p$ menor a 0,05 .

\section{Resultados}

De 900 pacientes estudiados, 36 (4\%) presentaron filtración.

De los 36 pacientes con filtración, 25 fueron mujeres y 11 hombres, relación hombre/mujer 1:2,2. Edad promedio 45,2 años e IMC preoperatorio $44,0 \mathrm{~kg} / \mathrm{m}^{2}$.

Fallecieron $3(0,33 \%)$ de 900 pacientes; $8,3 \%$ de los 36 pacientes con filtración. Uno con filtración en anastomosis gastroyeyunal y 2 en sutura duodenal. Dos eran hiperobesos $\left(\right.$ IMC $\left.>50 \mathrm{~kg} / \mathrm{m}^{2}\right)$ y uno de 60 años (Tabla 1).

De los 36 pacientes con filtración, presentaron fiebre $83,3 \%$ (30 pacientes), taquicardia $58,3 \%$ y dolor abdominal 47,2\%. Derrame pleural izquierdo o bilateral, en 17 pacientes $(47,2 \%)$, manejados medicamente, sólo 3 con punción pleural. Incremento de proteína $\mathrm{C}$ reactiva en $80,5 \%$ y leucocitosis en $69,4 \%$ de los casos (Tabla 2).

Diagnóstico de filtraciones: en $52,7 \%$ (19 casos) por estudios imagenológicos y $47,3 \%$ restante por salida de fluidos y/o azul de metileno por drenajes. Catorce pacientes diagnosticados por Tomografía Axial Computarizada (TAC) de abdomen; en $2^{\circ}$ estudio con bario, se comprobó filtración en 9 y cierre de filtración en 5. Un paciente presentó salida de jalea por drenaje y otro azul de metileno. El paciente con fluido por herida operatoria, presentó filtración de muñón yeyunal (Tabla 3 ).

Desde la operación 35 pacientes tuvieron drenajes, excepto uno. Todos dren izquierdo adyacente a anastomosis gastroyeyunal y 22 también al muñón duodenal. La Figura 4 muestra débito diario drenes hasta $30^{\circ} \mathrm{DPO}, \mathrm{y}$ resultados de un estudio previamente
Tabla 1. Características y mortalidad postoperatoria en 900 pacientes sometidos a BPGR

\begin{tabular}{|lllc|}
\hline Variables & $\begin{array}{l}\text { Sin filtración } \\
\mathbf{n}=\mathbf{8 6 4}(\mathbf{9 6 \% )})\end{array}$ & $\begin{array}{l}\text { Con filtración } \\
\mathbf{n}=\mathbf{3 6}(\mathbf{4 \%})\end{array}$ & $\mathbf{p}$ \\
$\begin{array}{l}\text { Género } \\
\text { - Femenino }\end{array}$ & 673 & 25 & n.s. \\
- Masculino & 191 & 11 & \\
Relación M/F & $1 / 3,5$ & $1 / 2,2$ & \\
Edad (promedio, DE, rango) & $40,6 \pm 12$ años & $45,2 \pm 11,5$ años & 0,024 \\
& $(14-64$ años) & $(23-65$ años) & \\
IMC preoperatorio $\left(\mathrm{kg} / \mathrm{m}^{2}\right)$ & $43,4 \pm 5,6$ & $44,0 \pm 7,1$ & n.s. \\
(promedio, DE, rango) & $(35-64)$ & $(35-67,4)$ & \\
Mortalidad: & $\begin{array}{l}\text { Fallecieron 3* }(0,33 \%) \text { del total de } 900 \\
\text { pacientes; 8,3\% de los 36 pacientes con }\end{array}$ & & \\
\hline
\end{tabular}

*1 con filtración gastroyeyunal y 2 con filtración duodenal.

Tabla 2. Presentación clínica en 36 pacientes con filtración después de BPGR

\begin{tabular}{|lccc|}
\hline $\begin{array}{l}\text { Síntomas, signos y } \\
\text { examen laboratorio }\end{array}$ & $\begin{array}{c}\text { Promedio } \pm \mathbf{D E} \\
\text { (rango) }\end{array}$ & $\begin{array}{c}\mathbf{N}^{\mathbf{0}} \text { de pacientes } \\
\mathbf{n}(\%)\end{array}$ & $\begin{array}{c}\text { Presentación } \\
\text { Día PO }\end{array}$ \\
$\begin{array}{l}\text { Fiebre }{ }^{\circ} \mathrm{C} \\
\left(>37,1^{\circ} \mathrm{C}\right)\end{array}$ & $\begin{array}{c}38,4 \pm 0,6 \\
(37,2-40,0)\end{array}$ & $30(83,3)$ & $9,3 \pm 10,5$ \\
$\begin{array}{l}\text { Taquicardia } \\
(\geq 100 \text { lat/min) }\end{array}$ & $\begin{array}{c}118,4 \pm 11,4 \\
(100-140)\end{array}$ & $21(58,3)$ & $10,9 \pm 13,8$ \\
$\begin{array}{l}\text { Dolor abdominal } \\
\text { Derrame pleural }\end{array}$ & - & $17(47,2)$ & $8,3 \pm 12,5$ \\
\hline $\begin{array}{l}\text { Proteína C reactiva } \uparrow \\
(\geq 10 \mathrm{mg} / \mathrm{l})\end{array}$ & $\begin{array}{c}257 \pm 124 \\
(83-549)\end{array}$ & $29(80,5)$ & $13 \pm 14,3$ \\
$\begin{array}{l}\text { Leucocitosis } \\
\left(>10.000 / \mathrm{mm}^{3}\right)\end{array}$ & $\begin{array}{c} \\
(10.672 \pm 4.450\end{array}$ & $25(69,4)$ & $11,6 \pm 12$ \\
\hline
\end{tabular}

$\mathrm{PO}=$ postoperatorio.

Tabla 3. Diagnóstico de filtraciones en 36 pacientes sometidos a BPGR

\begin{tabular}{|lccc|}
\hline Método diagnóstico filtración & $\begin{array}{c}\mathbf{n} \\
\text { pacientes }\end{array}$ & $\%$ & $\begin{array}{c}\text { Día PO del diag- } \\
\text { nóstico (promedio) }\end{array}$ \\
$\begin{array}{l}1^{\text {o }} \text { estudio radiológico GI con } \\
\text { Sulfato de bario }\end{array}$ & 5 & 13,9 & 5,4 \\
TAC abdomen y pelvis & 14 & 38,8 & 16,7 \\
Aumento secreción dren & 5 & 13,9 & 7,0 \\
Salida bilis por dren & 9 & 25,0 & 3,8 \\
Salida alimento por dren & 1 & 2,8 & 8,0 \\
Azul de metileno por dren & 1 & 2,8 & 16,0 \\
\hline $\begin{array}{l}\text { Salida de secreción por herida } \\
\text { quirúrgica }\end{array}$ & 1 & 2,8 & 13,0 \\
\hline
\end{tabular}

TAC $=$ Tomografía axial computarizada. 


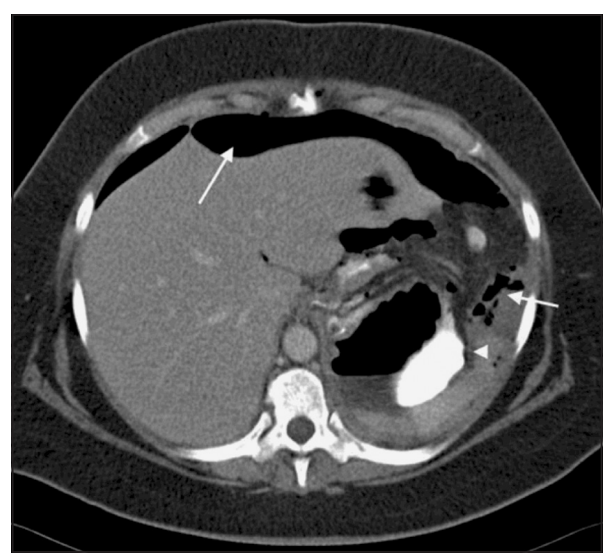

Figura 1. TAC de abdomen y pelvis: Filtración y neumoperitoneo.

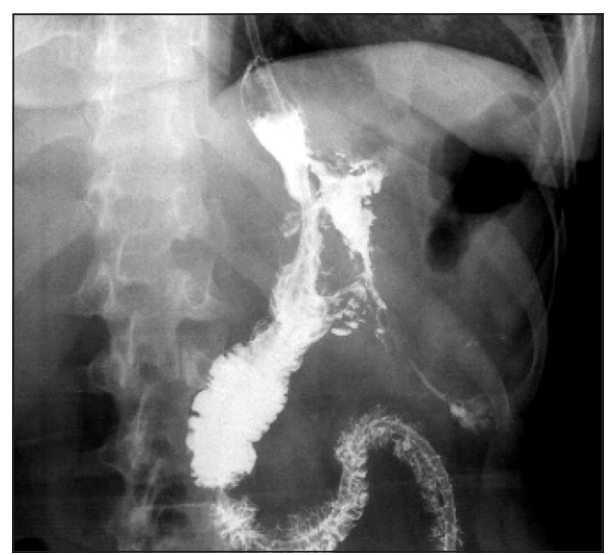

Figura 2. Filtración anastomosis gastroyeyunal control radiológico PO con bario.

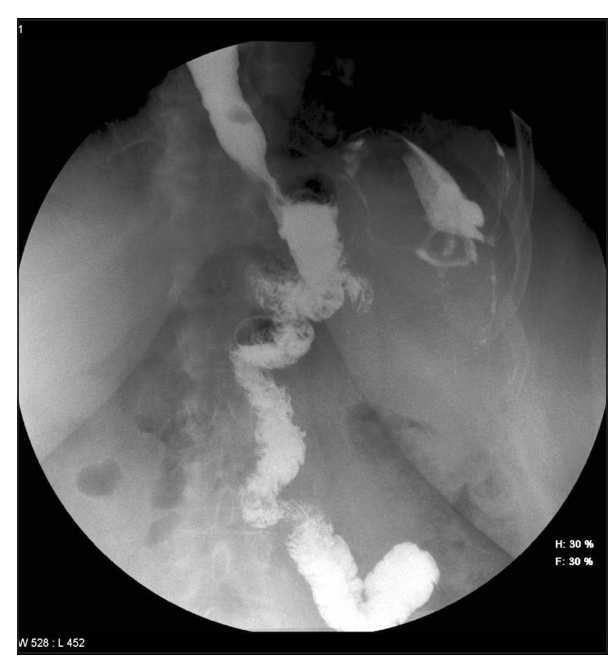

Figura 3. Filtración anastomosis gastroyeyunal control radiológico $\mathrm{PO}$ con bario. publicado $^{11}$. Se retiraron los drenes con fluido diario aproximadamente de $20 \mathrm{ml}$, prolongándose hasta el 52 DPO (Tabla 4).

Tiempo promedio diagnóstico 10,2 días, rango 1-59 días (Tabla 5).

Mayoría de las filtraciones entre $5^{\circ}-9^{\circ}$ DPO (Tabla 6).

Las filtraciones más frecuentes $(58,3 \%)$ fueron en anastomosis gastroyeyunal (Figura 5 y Tabla 7).

Según forma de presentación, predominaron las filtraciones tipo II, 91,7\% (Tabla 8).

El tratamiento fue quirúrgico en 12 casos (33,3\%). De los 12 pacientes operados, 4 fueron filtraciones tempranas, 3 intermedias y 5 tardías (Tabla 9 y Figura 6).

Período de tiempo entre diagnóstico y cierre de la filtración, varió entre 7 y 160 días, en promedio 35,5 días. Los períodos cortos tuvieron filtraciones subclínicas (Tabla 10).

El estudio microbiológico de las secreciones coleccionadas mostró ser variado y polimicrobiano (Tabla 11).

Tabla 4. Tiempo de retiro de drenes abdominales

\begin{tabular}{|llc|}
\hline Tiempo de retiro de drenes & $\begin{array}{c}\text { Pacientes } \\
\mathbf{n}=\mathbf{3 2 *}\end{array}$ & $\%$ \\
\hline $\begin{array}{l}\text { Entre } 5^{\circ} \text { y } 6^{\circ} \text { día PO } \\
(4 \text { reinstalación bajo TAC })\end{array}$ & 12 & 37,5 \\
- Entre $8^{\circ}$ y $12^{\circ}$ día PO & 5 & 15,6 \\
- Entre $13^{\circ}$ y $52^{\circ}$ día PO & 15 & 46,9 \\
\hline Día de retiro PO promedio: & $17,3(5-52)$ & - \\
\hline
\end{tabular}

*4 no tabulados: 1 sin dren, 2 reinstalados reoperación y 1 fallece sin retiro de dren.

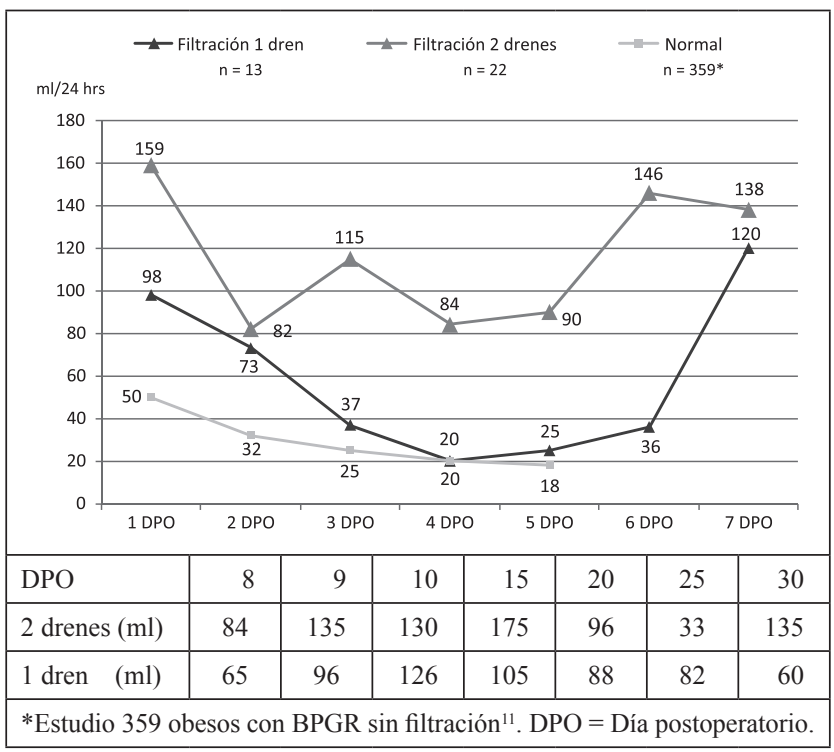

Figura 4. Débito diario de 1 ó 2 drenes en pacientes con filtración y con evolución normal*. 
Tabla 5. Tiempo de diagnóstico de filtraciones en 36 pacientes

\begin{tabular}{|c|c|}
\hline Tiempo de diagnóstico & n pacientes $(\%)$ \\
\hline 1 día PO & $3 \quad(8,3)$ \\
\hline 2 & $4(11,1)$ \\
\hline 4 & $1 \quad(2,8)$ \\
\hline 5 & $5 \quad(13,9)$ \\
\hline 6 & $3 \quad(8,3)$ \\
\hline 7 & $3 \quad(8,3)$ \\
\hline 8 & $2 \quad(5,6)$ \\
\hline 9 & $3 \quad(8,3)$ \\
\hline 10 & $1 \quad(2,8)$ \\
\hline 11 & $1 \quad(2,8)$ \\
\hline 13 & $2 \quad(5,6)$ \\
\hline 15 & $2 \quad(5,6)$ \\
\hline 16 & $2 \quad(5,6)$ \\
\hline 19 & $1 \quad(2,8)$ \\
\hline 23 & $1 \quad(2,8)$ \\
\hline 37 & $1 \quad(2,8)$ \\
\hline 59 & $1 \quad(2,8)$ \\
\hline \multicolumn{2}{|l|}{ Promedio $10,2 \pm 11$ día PO } \\
\hline \multicolumn{2}{|l|}{ Mediana 7 día PO } \\
\hline Rango $1-59$ día PO & \\
\hline
\end{tabular}

Tabla 6. Clasificación de filtraciones según tiempo de aparición

\begin{tabular}{|lcc|}
\hline Tipo filtración & $\begin{array}{c}\text { Pacientes } \\
\mathbf{n}=\mathbf{3 6}\end{array}$ & $\mathbf{\%}$ \\
\hline - Temprana $\left(1^{\circ}-4^{\circ}\right.$ día PO) & 8 & 22,2 \\
\hline - Intermedia $\left(5^{\circ}-9^{\circ}\right.$ día PO) & 16 & 44,5 \\
\hline - Tardía (10 0 o más día PO) & 12 & 33,3 \\
\hline
\end{tabular}

Tabla 8. Tipo de filtración según la severidad clínica

\begin{tabular}{|lcc|}
\hline Tipo filtración & $\begin{array}{c}\text { Pacientes } \\
\mathbf{n}=\mathbf{3 6}\end{array}$ & $\mathbf{\%}$ \\
\hline Tipo I (subclínica localizada) & 3 & 8,3 \\
\hline Tipo II (clínica diseminada) & 33 & 91,7 \\
\hline
\end{tabular}

Figura 5. Localización de las filtraciones. 1: anastomosis gastroyeyunal, 2: reservorio gástrico, 3: muñón yeyunal, 4: anastomosis yeyuno-yeyunal, 5: muñón duodenal.

Tabla 7. Localización de las filtraciones

\begin{tabular}{|lcc|}
\hline Localización filtración & $\begin{array}{c}\text { n pacientes } \\
\mathbf{n}=\mathbf{3 6}\end{array}$ & $\mathbf{\%}$ \\
\hline Anastomosis gastroyeyunal $(\mathrm{GY})$ & 21 & 58,3 \\
Reservorio gástrico & 3 & 8,3 \\
Muñón yeyunal & 1 & 2,8 \\
\hline Anastomosis yeyuno-yeyunal & 1 & 2,8 \\
Muñón duodenal & 7 & 19,4 \\
Doble filtración & 3 & 8,4 \\
- Anastomosis GY y duodenal & -2 & - \\
- Anastomosis yeyuno-yeyunal y duodenal & -1 & - \\
\hline
\end{tabular}

Tabla 9. Tratamiento en 36 pacientes con filtraciones

\begin{tabular}{|lcc|}
\hline Tratamiento & $\begin{array}{c}\text { Pacientes } \\
\mathbf{n}=\mathbf{3 6}\end{array}$ & $\mathbf{\%}$ \\
\hline $\begin{array}{l}\text { Quirúrgico (re-operación) } \\
\text { - Laparotomía, aseo y drenaje }\end{array}$ & 12 & 33,3 \\
- Sutura yeyunal & 6 & \\
- Sutura reservorio gástrico & 2 & \\
- Sutura duodenal, duodenostomía & 1 & \\
- Reoperación por evisceración (posterior & 1 & \\
presentaron filtración manejada con drenaje) & 2 & \\
Médico & 24 & 66,7 \\
- Mantención de drenes & 12 & \\
- Re-colocación drenes bajo TAC abdomen y & & \\
pelvis & 7 & \\
- Soporte nutricional, antibióticos & 3 & \\
- Sello con cianocrilato & 2 & \\
\hline
\end{tabular}




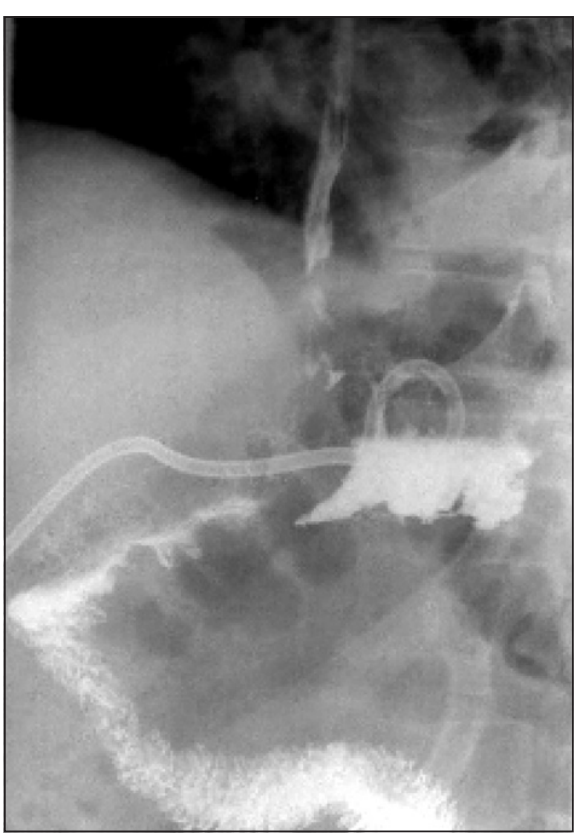

Figura 6. Drenaje percutáneo bajo TAC, para tratamiento de la colección intraabdominal posterior a la filtración gastroyeyunal

Tabla 11. Resultado microbiológico obtenido de cultivos de colecciones intraabdominales

\begin{tabular}{|c|c|}
\hline Estudio Microbiológico* $n=21$ & $\begin{array}{l}\text { Pacien- } \\
\text { tes }\end{array}$ \\
\hline Gram Positivos & $\mathrm{n}=12$ \\
\hline - Enterococcus sp & 4 \\
\hline - Enterococcus faecium & 1 \\
\hline - Enterococcus faecalis & 1 \\
\hline - Streptococcus alfa hemolítico grupo viridans & 3 \\
\hline - Streptococcus grupo viridans anginosus & 1 \\
\hline - Streptococcus beta hemolítico grupo c & 1 \\
\hline - Staphylococcus coagulasa negativa & 1 \\
\hline Gram negativos & $\mathrm{n}=8$ \\
\hline - Klebsiellla pneumoniae & 3 \\
\hline - Klebsiellla oxytoca & 1 \\
\hline - Pseudomonas aeruginosa & 1 \\
\hline - Escherichia coli & 2 \\
\hline $\begin{array}{l}\text { - Escherichia coli betalactamasa espectro ex- } \\
\text { pandido }\end{array}$ & 1 \\
\hline Hongos & $\mathrm{n}=1$ \\
\hline - Candida Albicans & 1 \\
\hline
\end{tabular}

* En los cultivos se aisló más de 1 bacteria.
Tabla 10. Período de tiempo entre diagnóstico y cierre de las filtraciones

\begin{tabular}{|lcc|}
\hline Duración de filtración & $\begin{array}{c}\text { Pacientes } \\
\mathbf{n}=\mathbf{3 2}\end{array}$ & $\mathbf{\%}$ \\
\hline 7 días & 1 & 3,1 \\
\hline $8^{* *}$ & 2 & 6,3 \\
$10^{* *}$ & 2 & 6,3 \\
$12 * *$ & 1 & 3,1 \\
14 & 2 & 6,3 \\
15 & 2 & 6,3 \\
17 - 21 & 4 & 12,5 \\
$22-27$ & 5 & 15,6 \\
$28-37$ & 6 & 18,7 \\
$44-97$ & 4 & 12,5 \\
$100-160$ & 3 & 9,3 \\
\hline Promedio \pm DE: & $35,5 \pm 38,0$ días \\
Mediana: & 22 días \\
Rango: & $7-160$ días \\
\hline
\end{tabular}

*No incluidos 3 pacientes fallecidos y 1 con cierre quirúrgico de la filtración. **Un paciente con filtración tipo I subclínica.

Reingreso hospitalario se produjo en $55,8 \%$ de pacientes con filtración (Tabla 12).

Se propone un algoritmo de manejo en filtraciones post BPGR (Figura 7).

\section{Discusión}

El bypass gástrico obtiene una baja de peso adecuada en obesos mórbidos, pero las complicaciones también pueden ser importantes, entre ellas la filtración.

La filtración puede o no comprometer la línea de sutura misma. Esta filtración puede producirse por deterioro o falla del cierre relacionado con la sutura empleada, sea sintética o metálica (engrapadora), denominándose dehiscencia a cualquier ruptura o abertura de la sutura quirúrgica, implicando alteraciones en el proceso de cicatrización normal. Factores de riesgo locales para una cicatrización alterada, incluyen inadecuada irrigación sanguínea e isquemia, infección y tensión exagerada en la sutura. La filtración también puede producirse por lesión del tejido adyacente a esta sutura en base a diferentes mecanismos como isquemia del tejido por lesión térmica (electrocauterio, LigaSure, etc). Desafortunadamente en estudios publicados sobre este tópico no se describe con qué frecuencia la filtración implica o no la línea de sutura ${ }^{12}$. Higa y cols ${ }^{13}$, reportaron en 400 pacientes con BPG laparoscópico, falla en 
Tabla 12. Estadía hospitalaria en 36 pacientes con filtración postoperatoria

\begin{tabular}{|lcc|}
\hline Estadía hospitalaria & $\begin{array}{c}\text { Pacientes } \\
\mathbf{n = 3 6}\end{array}$ & Días hospitalización (Promedio \pm DS rango) \\
\hline $\begin{array}{l}\text { Hospitalización total } \\
\text { - } 1^{\circ} \text { hospitalización }\end{array}$ & $36(100 \%)$ & $33,7 \pm 18,5(6-97)$ \\
$-2^{\circ}$ hospitalización (reingresos) & $19(55,8 \%)$ & $19,8 \pm 13,3(6-53)$ \\
\hline
\end{tabular}

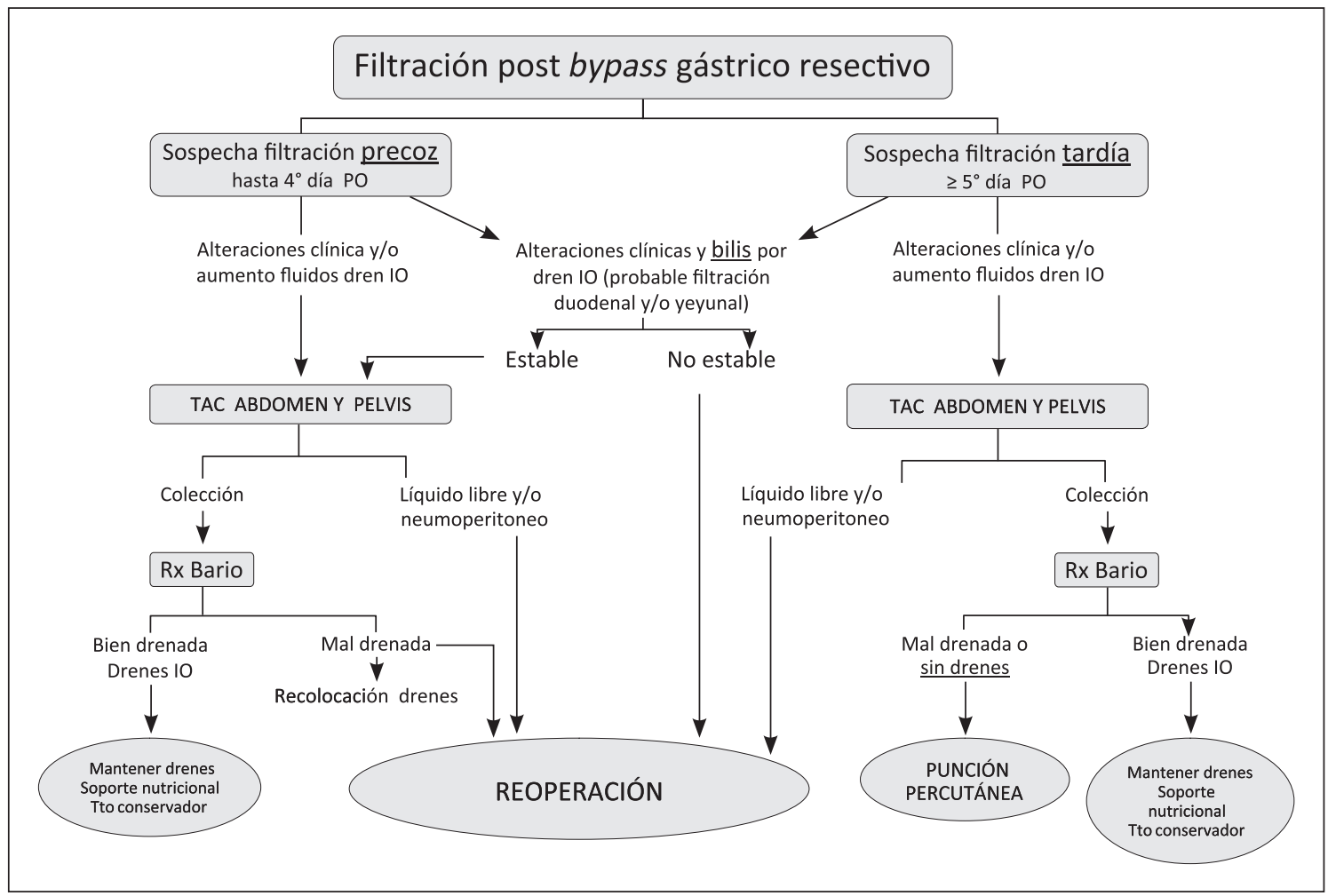

Figura 7. Algoritmo de manejo en filtraciones post bypass gástrico resectivo. $\mathrm{IO}=$ intraoperatorio.

la línea de corchetes en 6 pacientes, detectada en el intraoperatorio o 12 a 24 horas después de la cirugía, atribuyéndose al uso de una engrapadora.

Los sinónimos empleados para describir este evento son falla de anastomosis, insuficiencia anastomótica, disrupción de línea de sutura ${ }^{6}$. El término fístula hay que diferenciarlo ya que implica una comunicación anormal entre un epitelio y otra superficie epitelial. La filtración inicial de un órgano puede llevar posteriormente a la formación de una fístula o colección.

La incidencia de filtración en nuestra casuística fue $4 \%$. Reportes internacionales de BPG abierto mencionan cifras entre 0 a $5,6 \% \%^{8}$. Un estudio chileno multicéntrico muestra incidencia promedio de filtración de $1,3 \%$ después de BPG abierto y $1,4 \%$ después de BPG laparoscópico ${ }^{14}$.
Existen factores que incrementarían el riesgo de complicaciones y muerte en pacientes bariátricos. Mason y cols ${ }^{15}$, encontraron que la edad, mayor IMC y género masculino se asociaban con más complicaciones; igualmente Livingston y cols ${ }^{16}$, reportan una mortalidad 3 veces mayor en pacientes mayores de 55 años, comparándolos con los de menor edad, por falta de reserva en la recuperación de las complicaciones. Por otro lado Schwartz y cols ${ }^{17}$, describen mayor incidencia de filtraciones, hasta $20 \%$, en reoperados por fallas técnicas o inadecuada baja de peso. En nuestro estudio no hubo diferencia significativa en el género, pero la edad promedio fue mayor en el grupo con filtraciones. De los 3 fallecidos, 2 eran hiperobesos y 1 de 60 años. La mortalidad global de $0,33 \%$ fue similar a otros estudios, con rango 0,14 hasta $1,7^{14,18}$. 
Bellorin y cols ${ }^{19}$, correlacionaron las filtraciones que presentaron 8 de 518 pacientes con BPG laparoscópico con taquicardia de hasta 120 latidos por minuto 20 horas después de la cirugía en 5 de los 8 casos $(62,5 \%)$. En otro estudio, Hamilton y $\operatorname{cols}^{20}$, describen 9 filtraciones en 210 pacientes con BPG laparoscópico, concluyendo que el distress respiratorio y la taquicardia $>120$ latidos por minuto podían ser los indicadores clínicos más útiles de filtración. En nuestros pacientes que filtraron, la fiebre fue el signo más frecuente $(83,3 \%)$, siguiendo taquicardia con 118 latidos por minuto promedio. Hubo derrame pleural en $47,2 \%$ de los pacientes. Los conductos linfáticos del diafragma conducen líquido peritoneal, bacterias, y mediadores inflamatorios hacia la circulación sistémica a través del conducto torácico, pudiéndo esta conexión explicar la rápida afectación pulmonar observada en colecciones intraabdominales producidas por filtración. En presencia de colecciones subfrénicas, la mecánica respiratoria condiciona que la circulación del líquido intraabdominal se dirija hacia los espacios subdiafragmáticos.

Ante la sospecha de filtración por sintomatología clínica, la radiografia gastrointestinal con bario y el TAC son estudios que nos permitirán confirmar el diagnóstico. El diagnóstico de filtraciones se realizó con estudio radiológico de bario rutinario $(13,9 \%)$ y con TAC de abdomen y pelvis $(38,8 \%)$, mostrándose colecciones hidroaéreas adyacentes a la filtración y/o salida del contraste. De los 32 pacientes con estudio de bario entre $4^{\circ}$ y $5^{\circ}$ día PO, se diagnosticó filtración sólo en 5 , probablemente por aparición más tardía de la filtración. Un paciente $(2,8 \%)$ presentó fístula enterocutánea hacia herida operatoria. El tipo y volumen de fluidos por drenajes permitió el diagnóstico de filtración en el resto de pacientes (44,5\%), (Tabla 3, Figuras 1, 2 y 3 ).

Existen diferentes posturas respecto al control radiológico postoperatorio y probabilidad para detectar filtraciones, Schiesser y cols, comparan 2 grupos de pacientes con BPG laparoscópico: grupo A (382 pacientes), con radiografía GI con Gastrografin ${ }^{\circledR}$ entre el $3^{\circ}$ y $5^{\circ}$ DPO detectando 2 de 4 filtraciones en este grupo (sensibilidad de 50\%) ; grupo B (422 pacientes) con radiografía GI sólo en pacientes con sospecha clínica de filtración, detectando 4 de 5 filtraciones (sensibilidad 80\%), concluyendo que la radiografía GI era de valor limitado para la detección de la filtración ${ }^{21}$. Sin embargo, nos parece que el estudio con sulfato de bario permite demostrar la filtración con mayor claridad que el medio hidrosoluble.

Existe controversia en relación al drenaje profiláctico en cirugía gástrica ${ }^{22}$, nosotros dejamos drenes tubulares, adyacentes al muñón duodenal y anastomosis gastroyeyunal para detectar y/o tratar una filtración. En la Figura 4 observamos que las curvas de débito con 1 ó 2 drenes inician un descenso progresivo desde el día de la operación hasta el $3^{\circ}$ día postoperatorio, y posteriormente inician un ascenso; esta curva difiere de la presentada en el estudio de Csendes ${ }^{11}$, en la que desciende en forma permanente hasta medir $20 \mathrm{ml}$ y ser retirados los drenes.

La Tabla 4, muestra retiro de drenes promedio entre 5 a 6 días cuando inicialmente no se evidenció filtración, mientras que el retiro fue posterior cuando actuaron como tratamiento drenando líquido acumulado y se retiraron al cesar la producción.

Muchos cirujanos bariátricos usan drenes intraabdominales profilácticamente mientras que otros creen que no son de utilidad e incluso que favorecerían las filtraciones ${ }^{23}$. Salgado y $\operatorname{cols}^{24}$, evaluaron la respuesta inflamatoria peritoneal en presencia de un dren dejado hasta $7^{\circ}$ día posterior a BPG. El contenido del drenaje mostró incremento en niveles de factor de necrosis tumoral alfa e interleucina 1beta, aún en pacientes sin complicación intraabdominal, atribuyéndose al uso del dren por 7 días.

¿En qué día postoperatorio se producen las filtraciones con mayor frecuencia? Determinarlo nos permite estar más alerta para la detección, e incluso predecir la evolución, pues hemos observado que las filtraciones tardías tienen mejor evolución y cierran generalmente sólo con tratamiento médico, mientras que las tempranas ameritan muchas veces reoperación inmediata. Por esto tabulamos las filtraciones según período de tiempo de diagnóstico (Tabla 5) y según tiempo de aparición (Tabla 6), observándose ser más frecuentes las intermedias entre $5^{\circ}$ a $9^{\circ}$ día postoperatorio, con tiempo de aparición promedio de 10,2 días. Shiesser y cols, reportan tiempo promedio de diagnóstico de filtraciones al $5^{\circ}$ DPO en el grupo A y 4,25 DPO en el grupo B, rango $2-9^{\circ} \mathrm{DPO}^{21}$. A diferencia de Bellorin y cols, con 8 filtraciones, detectadas 6 al $1^{\circ}$ DPO y 2 al $6^{\circ}$ DPO, en 518 pacientes operados de BPG laparoscópico ${ }^{19}$.

Las filtraciones ocurren en diversos sitios de anastomosis o cierre de muñones en esta cirugía (Figura 5). El sitio más frecuente de filtración fue la anastomosis gastroyeyunal (58,3\%), seguido del muñón duodenal $(19,4 \%)$. Bellorin y cols ${ }^{19}$, informan 4 de 8 filtraciones $(50 \%)$ en anastomosis gastroyeyunal. En la serie nacional de Escalona, 641 pacientes con BPG no resectivo laparoscópico, predominan 5 filtraciones en la sutura vertical de la bolsa gástrica y 1 en la anastomosis gastroyeyunal ${ }^{25}$.

La evolución de las filtraciones concordó con la clasificación de severidad clínica, 3 pacientes con filtración tipo I, evolucionaron favorablemente en un período corto hasta el cierre de la filtración.

El manejo de una filtración depende de la magnitud en la abertura del tejido, de la cantidad y 
diseminación del líquido extraluminal y de la repercusión clínica que producen todos estos eventos en el tiempo. Las opciones de tratamiento incluyen tratamiento conservador con o sin drenaje externo y cirugía.

En las colecciones intraabdominales, hay que mantener los drenajes perianastomóticos y si no los hubiera, el drenaje percutáneo guiado con TAC es necesario ${ }^{26}$ (Figura 6).

El tratamiento quirúrgico vía abierta es realizado en pacientes con compromiso sistémico hemodinámico severo y sepsis, ocasionado por filtraciones tempranas, hasta 36 horas postoperatorias, o en pacientes que presentan colecciones intraabdominales no susceptibles de ser drenadas por otros procedimientos.

En nuestro estudio el tratamiento de las filtraciones fue principalmente médico, realizándose cirugía en 1/3 de los pacientes. La laparotomía exploradora, aseo de cavidad abdominal y drenaje fueron los procedimientos más utilizados. El tratamiento médico consistió en mantención o recolocación de drenes bajo TAC, soporte nutricional mediante alimentación parenteral o enteral y antibioticoterapia. Un tratamiento innovador como sello con cianocrilato fue empleado en 2 pacientes (Tabla 9).

El apoyo nutricional es fundamental para cubrir las necesidades del estado patológico del paciente ${ }^{27}$. Diagnosticada la filtración, el elemento central es evitar el contacto de nutrientes con el orificio de la filtración, por lo que se retrasa la alimentación oral algunos días, indicándose soporte nutricional por vía enteral por medio de sonda nasoenteral con su extremo distal posterior a la zona de filtración. Algunos pacientes requieren nutrición parenteral complementaria por vía periférica o central. El soporte nutricional se mantiene hasta el cierre de la filtración, siendo el aporte de nutrientes entre 25 a $30 \mathrm{cal} / \mathrm{kg} /$ día y $1,5 \mathrm{~g} / \mathrm{kg} /$ día de proteínas.

En 21 pacientes con estudio microbiológico de colecciones, todos los cultivos fueron polimicrobianos, reforzándose el concepto de sinergia bacteriana necesaria para producción del absceso, ya que los componentes de la flora mixta se complementan y potencian, tal como describe Parrilla ${ }^{28}$.

El espectro de patógenos en infecciones intraabdominales es diverso y depende del origen de la infección. Los patógenos aislados fueron principalmente Gram positivos, seguidos por Gram negativos y 1 caso Candida albicans. El estreptococo alfa hemolítico es parte de la flora oral gástrica. El esófago, estómago e intestino delgado proximal albergan flora de la saliva, que normalmente presentan densidad de bacterias muy baja, por esto al producirse perforación gástrica el grado de contaminación peritoneal es mínimo, sin embargo, con antiácidos, hemorragia, gastritis u obstrucción, se origina aumento en la densidad de bacterias. En nuestro estudio, en 3 pacientes se aisló estreptococo alfa hemolítico grupo viridans y en otro estreptococo anginosus grupo viridans, organismo identificado en el tracto gastrointestinal y asociado a infecciones purulentas invasoras ${ }^{29}$. Enterococos, bacterias gram positivas, se aislaron en 6 pacientes. En Chile, Enterococcus spp está entre las principales causas de infección nosocomial, asimismo Enterococcus faecalis y E. $_{\text {faecium }}^{30}$.

Sugerimos un algortimo de manejo de filtraciones post bypass gástrico resectivo y concluimos que, fiebre y taquicardia son signos útiles para sospechar filtración, siendo determinante el diagnóstico precoz de estas filtraciones, para lograr una mejor evolución del paciente y éxito en el tratamiento. El tratamiento médico con mantención o recolocación de drenes, soporte nutricional y antibioticoterapia es efectivo en la resolución de las filtraciones, ameritando reoperación sólo cuando éstas son tempranas y/o con evolución tórpida.

\section{Referencias}

1. Sugerman HJ, Starkey JV, Birkenhauer R. A randomized prospective trial of gastric bypass versus vertical banded gastroplasty for morbid obesity and their effects on sweets versus non sweets eaters. Ann Surg. 1987;205:613-24.

2. Brolin RE. Gastric bypass. Surg Clin North Am. 2001;81:1077-97.

3. Buchwald H, Williams S. Bariatric surgery worldwide 2003. Obes Surg. 2004;14:1157-64.

4. Csendes A, Burdiles P, Díaz JC, Maluenda F, Burgos AM, Recio M, y cols. Resultados del tratamiento quirúrgico de la obesidad mórbida. Análisis de 180 pacientes. Rev Chil Cir. 2002;54:3-9.

5. Csendes A, Burdiles P, Papapietro K, Díaz JC, Maluenda F, Burgos A, et al. Results of gastric bypass plus resection of the distal excluded gastric segment in patients with morbid obesity. J Gastrointest Surg. 2005;9:121-31.

6. Bruce J, Krukowski ZH, Al-Khairy G, Russell EM, Park KGM. Systematic review of the definition and measurement of anastomotic leak after gastrointestinal surgery. Br J Surg. 2001;88:1157-68.

7. Peel AL, Taylor EW. Proposed definitions for the audit of postoperative infection: a discussion paper. Surgical Infection Study Group. Ann R Coll Surg Engl. 1991;73:385-8.

8. Csendes A, Burdiles P, Burgos AM, Maluenda F, Diaz JC. Conservative management of anastomotic leaks after 557 open gastric bypasses. Obes Surg. 2005;15:1252-6. 
9. Csendes A. Conservative management of anastomotic leaks. Obes Surg. 2006;16:375-6.

10. Csendes A, Díaz JC, Burdiles P, Braghetto I, Maluenda F, Nava O, et al. Classification and treatment of anastomotic leakage after extended total gastrectomy in gastric carcinoma. Hepatogastroenterology 1990;37:174-7.

11. Csendes A, Burgos AM, Burdiles P, Maluenda F, Díaz JC, Rojas J. Uso de drenajes tubulares abdominales en pacientes con obesidad mórbida sometidos a bypass gástrico con gastrectomía. Rev Chil Cir. 2006;58:30-4.

12. Baker RS, Foote J, Kemmeter P, Brady R, Vroegop $\mathrm{T}$, Serveld M. The science of stapling and leaks. Obes Surg. 2004;14:1290-8.

13. Higa KD, Boone KB, Ho T, Davies OG. Laparoscopic Roux en Y gastric bypass for morbid obesity. Arch Surg. 2000;135:1029-34.

14. Csendes A, Maluenda F. Morbimortalidad de la cirugía bariátrica. Experiencia chilena en 10 instituciones de salud. Rev Chil Cir. 2006;58:208-12.

15. Mason EE, Renquist KE, Jiang DJ. Perioperative risks and safety of surgery for severe obesity. Am J Clin Nutr. 1992;55:573S-576S.

16. Livingston EH, Huerta S. Arthur D, Lee S, De Shields $\mathrm{S}$, Heber D. Male gender is a predictor of morbidity and age a predictor of mortality for patients undergoing gastric bypass surgery. Ann Surg. 2002;236:576-82.

17. Schwartz RW, Strodel WE, Simpson WS, Griffen WO. Gastric bypass revision: lessons learned from 920 cases. Surgery 1988;104: 806-12.

18. Birkmeyer NJ, Dimick JB, Share D, Hawasli A, English WJ, Genaw J, et al. Hospital complication rates with bariatric surgery in Michigan. JAMA. 2010;304:435-42.

19. Bellorin O, Abdemur A, Sucandy I, Szomstein S, Rosenthal RJ. Understanding the significance, reasons and patterns of abnormal vital signs after gastric bypass for morbid obesity. Obes Surg. 2011;21:707-13.

20. Hamilton EC, Sims TL, Hamilton TT, Mullican MA, Jones DB, Provost DA. Clinical predictors of leak after laparoscopic Roux-en Y gastric bypass for morbid obesity. Surg Endosc. 2003;17:679-84.
21. Shiesser M, Guber J, Wildi S, Guber I, Weber M, Muller MK. Utility of routine versus selective upper gastrointestinal series to detect anastomotic leaks after laparoscopic gastric bypass. Obes Surg. 2010;Sep 26 (Epub ahead of print).

22. Petrowsky H, Demartines N, Rousson V, Clavien PA. Evidence-based value of prophylactic drainage in gastrointestinal surgery: a systematic review and meta-analyses. Ann Surg. 2004;240:1074-84; discussion 1084-5.

23. Salgado W Jr, Macedo Neto MM, dos Santos JS, Sakarankutty AK, Ceneviva R, de Castro e Silva O Jr. Study of the patency of different peritoneal drains used prophylactically in bariatric surgery. World J Gastroenterol. 2009;15:2340-4.

24. Salgado W Jr, Cunha F de Q, dos Santos JS, NoninoBorges CB, Sankarankutty AK, de Castro e Silva O, et al. Routine abdominal drains after Roux-en-Y gastric bypass: a prospective evaluation of the inflammatory response. Surg Obes Relat Dis. 2010;6:648-52.

25. Escalona A. Complicaciones quirúrgicas en bypass gástrico laparoscópico. Rev Chil Cir. 2006;58:97-105.

26. Scheirey CD, Scholz FJ, Shah PC, Brams DM, Wong BB, Pedrosa M. Radiology of the laparoscopic Rouxen-Y Gastric Bypass procedure: conceptualization and precise interpretation of results. RadioGraphics 2006; 26:1355-71.

27. Papapietro, K. Asistencia nutricional en cáncer gástrico. En: Csendes A, Korn O, Burdiles P. Cáncer gástrico. Santiago: Editorial Mediterráneo; 2006:260-8.

28. Parrilla Paricio P, Landa García J. Infección en Cirugía. En: Cirugía AEC, $2^{\text {a }}$ ed. Madrid: Editorial Médica Panamericana; 2010: (1227 p.)

29. Whiley RA, Beighton D, Winstanley TG, Fraser HY, Hardie JM. Streptococcus intermedius, Streptococcus constellatus and Streptococcus milleri group: Association with different body sites and clinical infections. J Clin Microbiol. 1992;30:243-4.

30. Marovac J, Campos MI. Enterococcus faecium resistente a vancomicina. Rev Med Chile 2000;128:685-6.

\section{INFORME}

Agradezco al Directorio de la Sociedad de Cirujanos de Chile el haberme designado cirujano informante de la Dra. Ana María Burgos Li, en su trabajo de ingreso ya que de esta manera puedo cooperar con humildad a los postulados de nuestra sociedad y por otro lado dar testimonio del esfuerzo de la Dra. Burgos para estar exponiendo su trabajo de ingreso, más cuando día a día son menos los que quieren entrar a este seno quirúrgico con los requisitos tradicionales.

La Dra. Burgos de nacionalidad peruana, estudió medicina en la Universidad Nacional de Trujillo y luego realizó un postgrado en cirugía en la misma universidad. En su constante inquietud en el año 1997 hizo una estadía de Perfeccionamiento en el Hospital Clínico de la Universidad de Chile, J.J. Aguirre, donde siguió trabajando ad honoren en investigación, hasta cuando fue contratada en el Servicio de Cirugía en el año 2005. Cabe destacar que realizó su revalidación profesional en Chile y se certificó como Cirujano General en el CONACEM lo que demuestra su constancia y esfuerzo. 
En el presente trabajo la Dra. Burgos nos presenta la experiencia de 10 años de bypass gástrico resectivo laparotómico de dicho Hospital Clínico, enfocado a las filtraciones, una de las complicaciones más temidas por los cirujanos, lo que constituye un referente a nivel nacional por el gran número de cirugías presentadas.

Desde el punto de vista antedicho, resulta un trabajo que aporta datos valiosos para el diagnóstico y tratamiento de esta complicación. Es importante señalar que las clasificaciones utilizadas, tanto para el tiempo de presentación, localización y severidad de filtraciones en bypass gástrico, resultan un gran aporte, que permitiría realizar comparaciones más precisas entre series de bypass gástrico de diversos grupos quirúrgicos.

La experiencia expuesta en cuanto al manejo de las filtraciones, coincide con la tendencia actual hacia el manejo conservador; aprovechando los beneficios de la radiología intervencional.

$\mathrm{Si}$ bien es cierto, una incidencia de un $4 \%$ de filtraciones es un poco mayor a la experiencia nacional, tanto para bypass convencional abierto como laparoscópico de acuerdo a lo publicado por el Profesor Csendes en la Revista Chilena de Cirugía en el año 2006, ésta puede ser explicada porque esta experiencia se remonta desde el año 1999, con curva de aprendizaje y sin los avances de los instrumentos actuales.

Llama la atención que a pesar de la gran experiencia endoscópica del grupo quirúrgico y la tendencia al manejo conservador, no haya un solo paciente en el cual se utilizaron endoprótesis cuyo rol en complicaciones en cirugía bariátrica cobra cada vez más fuerza, y su uso es cada vez de más amplio consenso; por otro lado se utilizó cianocrilato, cuyo rol en filtraciones no está claro y la evidencia disponible no va más allá de reportes de casos.

El bypass gástrico resectivo agrega al bypass gástrico tradicional una complicación con alta mortalidad, como lo es la filtración del muñón duodenal, ya que de los 3 pacientes fallecidos reportados $(20 \%$ de mortalidad de los pacientes que filtraron el duodeno), 2 correspondían a este tipo de filtración. Sin embargo, no se le da el peso que corresponde en el presente trabajo y se considera como una más de las filtraciones descritas, cuyo pronóstico es francamente más benigno.

Probablemente el bypass resectivo tiene un rol limitado a pacientes con lesiones neoplásicas o lesiones preneoplásicas de alto riesgo (metaplasia intestinal moderada o severa y displasia) en el estudio preoperatorio y en pacientes con gran carga genética de cáncer gástrico y que tengan bajo riesgo quirúrgico.

En Chile, el cáncer gástrico es principalmente de tipo intestinal y endémico; secundario principalmente a factores ambientales, siendo el Helicobacter pylori el más importante en su variedad asiática.

Considerando la erradicación de H. pylori previo a un bypass gástrico y la exclusión de la alimentación del estómago remanente, teóricamente ese segmento tendría un muy bajo riesgo de cáncer gástrico. Incluso pudiera considerarse el bypass gástrico como un elemento protector de cáncer gástrico, lo cual se condice con los pocos casos reportados de cáncer de dicha localización en pacientes sometidos a bypass, siendo que es una técnica que tiene más de 40 años.

Es difícil extender las mismas conductas de manejo presentadas en esta serie, para filtraciones precoces, a la técnica laparoscópica; debido a que la reintervención por esta vía significa un impacto mucho menor que la vía laparotómica. Pacientes no necesariamente graves o con sepsis severa, también se ven beneficiados con una reintervención por vía laparoscópica, que implica aseo de la cavidad peritoneal, reposición o instalación de drenajes, eventual intento de cierre de la filtración con sutura manual, asistido por la instalación de endoprótesis.

Dr. Cristián Ovalle L. ${ }^{1,2,3}$ ${ }^{1}$ Clínica Tabancura. ${ }^{2}$ Hospital de la Fuerza Aérea de Chile. ${ }^{3}$ Facultad de Medicina Universidad de Valparaíso. Santiago, Chile. 